\title{
Research on the "Instantaneity" of Documentary Photography from the Perspective of Reception Aesthetics
}

\author{
Liu Fan ${ }^{1,}$ Zhang Yansong ${ }^{2, b^{*}}$ \\ 1. 2 School of Fashion, Dalian Polytechnic University, Dalian, Liaoning, China \\ a1546214272@qq.com \\ b*2446843325@qq.com
}

\begin{abstract}
Documentary photography mainly focuses on the photographer and the subject. However, in the contemporary all-media "image reading era" and "national photography era", the level of image readers is more complex and extensive, which means that the audience will be more engaged in the process of completing the picture, the reader's acceptance has played an important role in the completion of the photographic works. Through the interdisciplinary research on the reception aesthetics and documentary photography, the author takes Frank's American as an example and the documentary photography can be analyzed from a different perspective.
\end{abstract}

Keywords: reception aesthetic, documentary photography, aesthetic experience, indecisive moments

\section{接受美学视域下纪实摄影的“瞬间性”研究

$$
\text { 刘璠 }{ }^{1,} \text { a张岩松 }{ }^{2,} \text { b* }
$$

1,2 大连工业大学服装学院, 大连, 辽宁, 中国

a1546214272@qq.com

b*2446843325@qq.com

\section{摘要}

纪实摄影的着眼点大多集中在摄影师与拍摄对象上, 然而在今天这个全媒体“读图时代”以及“全民摄影时代”， 图片读者的层次更加复杂和广泛，这也就意味着观众将更多地参与到图片的完成过程中，读者的接受对于摄影 作品的完成起到了重要的作用，笔者通过对于接受美学和纪实摄影的跨学科研究，以弗兰克拍摄的《美国人》 为例, 进而可以从不一样的视角分析纪实摄影。

关键词: 接受美学; 纪实摄影; 审美体验; 非决定性僢间

\section{1. 前言}

接受美学诞生于二十世纪六七十年代的德国, 是 当时盛行的思潮之一, 其讨论的大部分内容都是围绕 着读者的接受状态与文本之间的关系展开, 随着不同 学科的跨界交融, 接受美学这一美学理论也在图像化 的艺术作品鉴赏和创作中加以阐释和讨论, 而摄影作 为当代艺术较为关注的艺术形式自然也加入了接受

\section{美学的讨论之中。}

\section{2. 接受美学理论}

接受美学在二十世纪六十年代德国的大环境中 酝酿而来，彼时德国的“经济奇迹”出现衰退，由此引 发了人们对于传统制度与思想的反思，与此同时二十 世纪六十年代中期的文学、美学在研究与发展上都出 现了困境，在这种困境之中汉斯·罗伯特·尧斯和沃尔 
夫冈·伊瑟尔两位率先走出了传统美学研究的困境, 不局限于作者与作品, 将关注点转移到了读者身上, 进而创立了接受美学理论。

尧斯认为读者对于文学作品的接受并不是完全 的被动, 反而在接受文学作品之前就已经形成了对于 文学作品的期待, 也就是尧斯所提出的“期待视野”这 一概念。“期待视野”包含三个方面, 一是对作品体裁 和标准的了解, 在阅读前可以分辨作品类型; 二是对 文学史的了解, 可以通过对于以往的作品记忆来阅读 新的作品; 三是读者自身的社会生活经验, 不自觉地 将现实生活与文本内容结合起来。在作品鉴赏和分析 的过程中, 将作者和作品的单向输出通过上述三个方 面改为读者的先觉预判, 从而激发起读者对作品内容 的期望与猜测。

作为接受美学的另一代表人物, 伊瑟尔则提出了 “召唤结构”这一理论, 召唤结构主要就是指文学作品 在没有观者的阅读之前是不完整的, 文中留有“空白” 的地方需要读者去填充, 也就是读者受到了文本的 “召唤”。在伊瑟尔的“召唤结构”中, 能够召唤读者的 是以下三个方面, 一方面是文章结构和内容上的“空 白”, 需要读者通过自己的理解和经验进行补充; 其 次是文本中打破常规, 挑战读者惯性思维的“否定 性”, 通过这样一种形式来打破观者已经形成的固有 思维方式, 重新审视作品; 最后则是文本中两个连续 情节或结构之间的连接“空缺”, 读者的阅读是连贯 的, 自然会将这些“空缺”填补上并在“空缺”中建立自 己的理解。

尧斯和伊瑟尔作为接受美学的代表人物, 他们摆 脱了传统文艺分析的束缚, 将读者作为完成作品的重 点, 使读者在结构性空白中填补空白并与文本相互作 用形成完整的作品, 读者在填充文本空白的同时, 也 赋予了作品新的意义一一种接受者视角的作品。这 样将接受者作为创作思考对象的思维方式, 拓宽了文 学艺术分析思路的同时, 也为其他类型的艺术创作带 来了更多可能。在具有作者、文本与读者的艺术形式 中, 我们可以用接受美学去分析和解释, 获得不一样 的审美体验, 这其中我们可以将目光从文字媒介转移 到图像媒介之上, 比如摄影艺术。

\section{3. 纪实摄影的困境与接受美学}

摄影术自 1839 年诞生以来, 就以其记录真实的 特性（纪实性）而闻名, 并以其纪实性在艺术领域站 稳了脚跟。纪实摄影作为摄影艺术中重要的分支之 一, 摄影师将相机做笔, 图像为字, 向观众展现自己 眼中的世界, 这与接受美学中的作者、文本和读者遥 相呼应, 同时纪实摄影作为摄影艺术中生命周期最长 的门类, 其评判标准百年来一直是瞬间的记录, 这无 疑给纪实摄影的发展造成了阻碍与困境, 因此我们可 以尝试用接受美学理论分析纪实摄影, 从新的角度审 视纪实摄影, 为纪实摄影解围。

纪实摄影诞生于 19 世纪末, 于 20 世纪半叶的美
国成长，其秉承着在拍摄作品的过程中，对事实进行 记录, 而不是以个人的情感或灵感表达为出发点进行 拍摄（部分纪实摄影在某些方面也可以传递情感）的 理念, 但摄影师在创作的时候难免会将主观情感加入 其中, 但正是如此纪实摄影美学才得以蓬勃发展, 出 现了多萝西娅·兰格、刘易斯·海因、罗伯特·卡帕和卡 蒂埃·布列松等一系列纪实摄影大师, 其中以布列松 和他所提出的“决定性瞬间”最为著名。

布列松将生活中最普通的瞬间通过自己对于世 界的充分把握以极高的美学标准记录下来: 将事物的 形式与内容、时间与空间这几个方面恰到好处地呈现 出来（如图 1、2)。但是他的画面处于一种“被填满” 的状态, 画中的一切元素都处于不可动摇的位置上, 我们作为观者能做的只有观察和欣赏这样的照片, 但 并没有参与到其中，拉开了观者与照片之间的距离, 在达到了美学高度的同时也限制了纪实摄影的更多 可能。

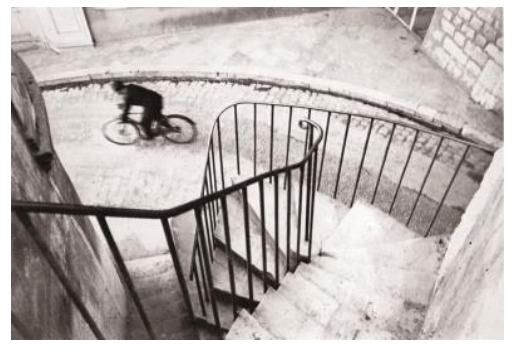

图 1 《雅尔》, 1932 年。（卡蒂埃・布列松 摄）

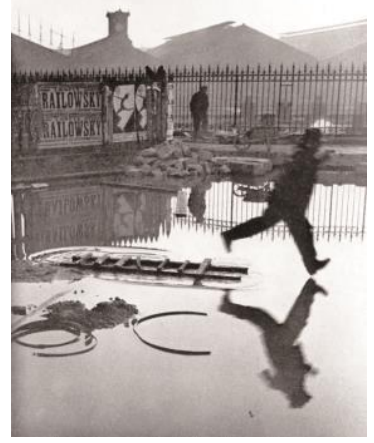

图 2《巴黎圣拉萨车站后方》, 1932 年。(卡蒂埃・布 列松 摄)

与文学、美学所处的困境相同, 此时的纪实摄影 由于百年来审美标准的固化, 并没有走出作者与作品 的局限, 接受美学的提出解决了文学、美学的困境, 而以罗伯特. 弗兰克为代表的摄影师提出了“非决定 性瞬间”, 以反叛者的角色重塑了纪实摄影的审美, 打破了纪实摄影“被填满”的局限, 留出了更多的“空 白”让观看者参与进来, 将关注点转移到了观众和普 通人的审美上。

\section{4. 接受美学与 “非决定性瞬间”}

“非决定性瞬间”诞生于 20 世纪中叶与接受美学 诞生时间相近，同时都是困境之中的出路与对传统的 
反思与反叛, 更重要的是二者在反叛的同时都将视线 从作者 (摄影师) 和文本 (照片) 之中脱离出来, 营 造了更多“文本空白”与读者进行互动, 读者的参与使 得作品跳脱了作者的单向输出, 转向了通过不同背景 的读者解读的多维互动, 将焦点集中在观看者身上, 同时在 “非决定性瞬间”中可以发现与接受美学相呼 应的“期待视野”与“召唤结构”。

从接受美学去分析“非决定性瞬间”则离不开这 一理论的代表人物罗伯特. 弗兰克和他拍摄的《美国 人》系列作品（如图 3)，他在《美国人》系列中的 场记基本都是人群集中的户外, 其中有街头游行、教 堂、公园、婚礼或者是商业活动场景。与布列松等知 名摄影大师的传统纪实摄影不同, 他聚焦于默默无闻 的人和平凡的场景, 弗兰克走过了美国的一个个城 市, 拍摄了那些各式各样不起眼的路人, 也有很多的 车, 还有无数的旗帜。从技术层面来看, 弗兰克一反 常规, 无论是构图、影调还是拍摄对象的选择似乎都 在以一种反叛的方式在创作; 从作品内涵上来看, 在 普通人看来 “英雄” 的美国反而成了颓废、孤独、不安 的大地。当时的艺术评论家认为他的照片不仅技术粗 糙, 像个外行人, 而且拍摄的内容将美国的繁荣发展、 人们的幸福美满无视, 像是一个反社会的刻薄记录 者, 因此有一些人将他贴上“反美”的标签。当时的一 些主流媒体譬如《时代》杂志和马格南图片社对《美 国人》的态度都不是很友好, 他们觉得这些粗婳的照 片应该被丢进垃圾桶; 美国的《大众摄影》甚至给弗 兰克扣上了“憎恶收留他的国家的人”的帽子。

但是, 好的艺术作品总是能经得起推敲, 好的艺 术家总是能预判时代和社会的发展, 所以时间证明了 一切, 10 年后, 也就是二十世纪六十年代, 与接受 美学诞生的时间和背景相同, 政治、经济、社会中的 一系列规则制度被打破, 新一代艺术家们开始认可弗 兰克, 《美国人》系列作品成为当时政治、社会、经 济、文化背景下美国人的焦虑、迷茫、不安、冷漠、 疏离的最好图像。

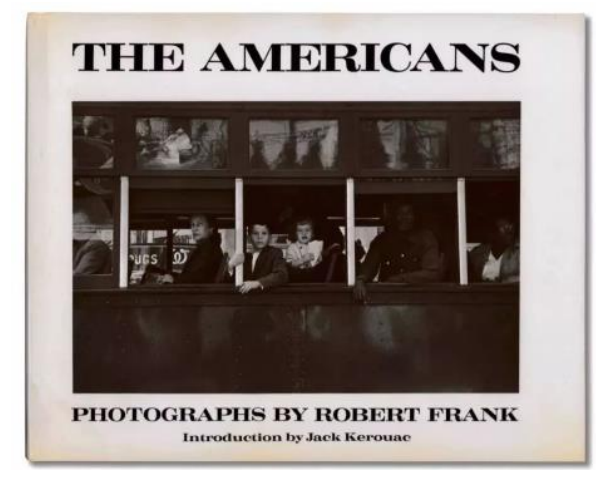

图 3 《美国人》影集, 1955 年。

从期待视野的角度来看, 这部影集读者对于作品 的期待有以下三个方面, 一是从选题上就选择了易于 大众接受的“美国人”这一主题, 使得每位观看者对于 主题都有一定的了解, 同时因为不同的文化背景和社
会经验, 可能会产生不同的理解与期待; 二来作为一 名在美国的瑞士人所拍摄的美国人这一主题, 这样的 趣味性也会引起读者的期待; 第三则是对于一直以来 主导人们纪实摄影审美思维的“决定性瞬间”，这一点 不仅是《美国人》的“期待摄影”也是“非决定性瞬间” 的“期待视野”。而正是因为有了以上这三种期待，使 得更多的观者参与进来, 与作品形成了互动。

从召唤结构的角度来分析, 其一是弗兰克拍摄的 《美国人》通过画面中的虚焦与粗粘的颗粒感营造出 了一种“文本空白”（如图 4、5），用模糊的对焦将画 面主体模糊, 看似不专业的手段实则为观者留下了想 象和补充的空间, 通过这种模拟人观察事物的局限性 (并不能抓取清晰的瞬间) 来拉近和观者的距离; 其 二弗兰克用看似随意无序的镜头，使得镜头之间割裂 开来，从而制造一种“文本空缺”，让读者阅读的连贯 性将情节连接, 并在“空缺”处建立自己的理解; 其三, 也是最关键的一点则是弗兰克利用倾斜变形的构图 和反审美的瞬间, 描绘了他眼中的美国和美国人: 充 满颓废、焦躁、消极和失望的气息, 人们的孤独, 与 城市的格格不入, 在巨大繁荣兴盛的表象之下, 是我 们看不见的真实, 正是这些期待与实际作品的不符, 使得《美国人》这部影集中出现了成为了一部“否定 性”的作品, 挑战了观者惯性认知, 让人们从新思考, 进而认识真实的世界。

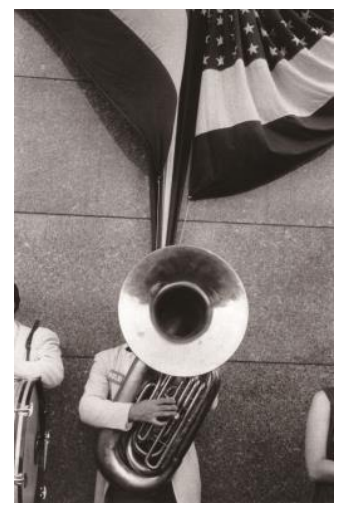

图 4 《美国人》之一, 1955 年。(罗伯特・弗兰克 摄)

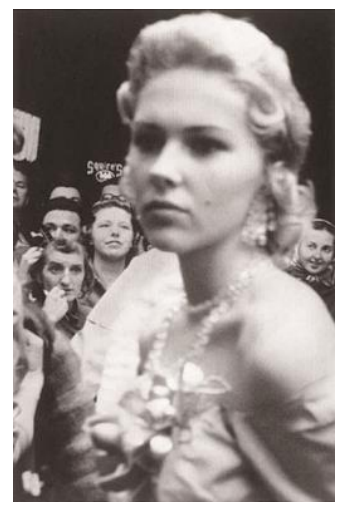

图 5 《美国人》之一, 1955 年。(罗伯特・弗兰克 摄)

从“期待视野”和“召唤结构”的视角分析“非决定 性瞬间”, 让我们获得了接受美学视角下的纪实摄影, 
“非决定性瞬间”打破传统纪实摄影审美, 将画面从完 美之中解放出来, 拉近了观者与作品之间的距离, 使 得观者对于摄影作品有了新的期待, 而作者在营造期 待的同时, 通过图像语言的空白、空缺和否定性, 让 观者参与进来的同时增加了与作品之间的互动, 继而 在图片否定性上引起观者的反思来达到观者的接受, 进入接受美学体验之中。

\section{5. 结论}

艺术的目的与功用在于使观赏主体的感觉“陌生 化”, 克服习惯的机械认知, 从而达到对事物内在生 命的直接体验, 纪实摄影亦是如此, 尧斯和伊瑟尔的 接受美学将我们对于摄影作品的欣赏从研究作者和 作品上解放出来, 让我们不受困于摄影技术并超越常 规固化的审美局限, 进而真正的感受到摄影作品中所 包含的真诚情感和深刻意蕴, 而尧斯的期待视野中提 到的不同文化背景和社会经验, 也让我们意识到了自 身的局限性, 因此我们无论在纪实摄影的拍摄还是分 析欣赏中, 都要从观看者的视角与作品形成互动, 超 越自身、技术以及审美的局限性, 复归于贞儿, 像一 个接受者一样去思考, 挣脱限制我们想象的“牢笼”, 以最原始的眼光与思维, 放空自己, 让自己达到一个 “无”的境界，将连贯性的生活打破、将思维而忽视的 问题将暴露出来, 从无到有再到无, 获得更多的“文 本空白”, 让我们具有成长突破的空间。

与此同时, 我们的眼光不应该局限于接受美学与 艺术作品的结合, 也许我们也可以通过对于接受美学 的分析, 进而对社会、生命有所感悟。在这个充斥着 消费主义的时代, 铺天盖地的广告乃至于主流价值观 大部分都是消费带来的快乐, 宣传的趋势也都是如果 你购买了, 你将拥有广告里一样的梦幻人生, 我认为 这是一个危险的趋势。它让我们活在“泡沫”之中, 在 “泡沫”的幻境之中得到满足, 而这样的“泡沫”却极易 破灭, 这就使得我们的承受力变低, 变得脆弱, 受制 于这些幻境, 让我们本该平静的内心世界变得焦躁起 来, 这种幻境麻痹着人们阻止人们去真正地改变现 状, 这是人类真正的不自由。如同上文所说, 我们要 把自己“重置”, 放空自己, 以接受者的视角打破现有 的局限, 跳出自我的虚假安全区, 将连贯的生活和思 维打破, 真诚无畏地接受世界和我们的生命。

\section{REFERENCES}

[1] Wang, T., Jia, W. (2020) Recreation of the aesthetic potential of literary texts under the theory of "response-inviting structure" by Iser. Journal of Hubei University of Education, 37(04): 7-11.

[2] Liu, S.Y. (2020)The breakthrough and predicament of the paradox of literary history-On Hans Robert Jauss 's view of literary history. Literary Controversy, (10): 97-102.

[3] Yang, J. (2014) The decisive moment and the indecisive moment. House of Drama (first half of the month), (05):214+219.

[4] Zeng, X.T. (2019) Reception Aesthetics in Art. Grand View of Fine Arts, (10):64-65.

[5] Gu, Z. (2006)World Photography History. Zhejiang Publishing House, Hangzhou. 61. 